\title{
Adaptive Balancing by Reactive Compensators of Three-Phase Linear Loads Supplied by Nonsinusoidal Voltage from Four-Wire Lines
}

\author{
Leszek Czarnecki, Motab Almousa \\ School of Electrical Engineering and Computer Science, Louisiana State University, Baton Rouge, USA
}

Email address:

Iczarn1@Isu.edu (L. Czarnecki),malmou2@Isu.edu (M. Almousa)

To cite this article:

Leszek Czarnecki, Motab Almousa. Adaptive Balancing by Reactive Compensators of Three-Phase Linear Loads Supplied by Nonsinusoidal Voltage from Four-Wire Lines. American Journal of Electrical Power and Energy Systems. Vol. 10, No. 3, 2021, pp. 32-42.

doi: $10.11648 /$ j.epes.20211003.11

Received: January 13, 2021; Accepted: February 23, 2021; Published: May 20, 2021

\begin{abstract}
A method of the design of an adaptive balancing reactive compensator in four-wire systems with linear loads and nonsinusoidal voltage is described in this article. The method of compensation is founded on the Currents' Physical Components (CPC) - based power theory of three-phase systems with nonsinusoidal voltages and currents. The compensator is built of two sub-compensators of $\mathrm{Y}$ and $\Delta$ structure, respectively. The $\mathrm{Y}$ compensator reduces the reactive current and the zero sequence symmetrical component of the unbalanced current. The $\Delta$ compensator reduces the negative sequence symmetrical component of the unbalanced current. The positive sequence symmetrical component of the unbalanced current and the scattered current remain uncompensated. It is because shunt reactive compensators do not have any capability for that. Thyristor Switched Inductors (TSIs) enable the susceptance control of the compensator branches, referred to in the article as Thyristor Controlled Susceptance (TCS) branches. Periodic switching of thyristors in these branches causes the generation of harmonic currents, in particular the third-order harmonic. Moreover, in the presence of the supply voltage harmonics, a resonance of the equivalent capacitance of the compensator with the distribution system inductance can occur. These two harmful phenomena in the compensator suggested were reduced by the selection of a special structure of the TCS branches and their $L C$ parameters. The presented method of the adaptive compensator synthesis was verified in the article with a numerical example and results of computer modeling of the load with an adaptive compensator.
\end{abstract}

Keywords: Asymmetrical Systems, CPC, Currents' Physical Components, Unbalanced Loads, Power Definitions

\section{Introduction}

Degradation of the power factor in large manufacturing plants is a combined effect of the reactive power, the load imbalance, and harmonic currents generated by nonlinear or periodically switched loads. Electrical loads in such manufacturing plants are both three- and single-phase ones. The load imbalance is caused by the presence of single-phase loads in such plants. Such imbalance causes that an unbalanced current occurs in the supply lines of such plants, causing asymmetry of the distribution voltage. Therefore, the distribution system has to be built as a three-phase system with a neutral conductor. Consequently, a compensator needed for the power factor improvement should have the capability of compensating not only the reactive and unbalanced currents but also the neutral conductor's current. It should have, moreover, adaptive property.

A low power factor and the current asymmetry have economic and technical consequences, Therefore, they are the subject of long-lasting studies, initiated by Steinmetz in 1917 [1].

This research on methods of compensation is continued now. It includes studies on compensation with static reactive LC compensators, with thyristor controlled compensators and with hybrid compensators, composed of a static LC compensator and thyristor-controlled reactors [2-6]. Taking into account the very high power of manufacturing plants, switching compensators, SC, commonly known as active power filters, built of transistors, may not have sufficient power for compensation of such very high power loads.

This article is the next step in studies on compensation in 
three-phase systems. These studies started with developing a power theory of three-phase, three-wire systems with nonsinusoidal voltages and currents, known now as the Currents' Physical Components (CPC) - based power theory (PT) [7]. This theory provided fundamentals for developing a method of design of reactive balancing compensators operating in the presence of the supply voltage distortion [8]. The CPC - based PT was extended for three-phase systems with a neutral conductor and nonsinusoidal supply voltage [9]. A method of synthesis of balancing reactive compensators for such systems was also developed by Czarnrcki [10]. Compensators as discussed in the above referenced articles were fixed-parameters devices, however. An approach to conversion into adaptive devices was suggested by Czarnrcki and Almousa in [11]. This article is a direct continuation of it, aimed at improvement of the previously obtained results. Since this article is built upon these results, it would be recommended that the reader, if needed, is acquainted with those details and results [7-11].

\section{The Approach to the Compensator Development}

The compensator under the development has to be controlled in real-time so that the time-consuming optimization approach to its design has to be excluded. Its parameters have to be specified by algebraic expressions. The method suggested includes the following five steps.

1. The LC parameters of a static reactive compensator, composed $\Delta$ and $\mathrm{Y}$ sub-compensators are calculated, based on the original method developed in the frame of the CPC - based power theory.

2. The complexity of the compensator branches is reduced to branches built of no more than two reactive elements by a branch.

3. The range of change of the reactive power and the load imbalance, as well as the voltage harmonics are evaluated and the range of changes of the compensator susceptances are calculated.

4. Branches of the static LC compensator are replaced by branches with thyristor-controlled susceptance (TCS).

5. A look-up table, that enables the selection of thyristor firing angle for needed susceptance of each of six TCS of the compensator is created.

The presented method of compensation was verified by a computer simulation (Matlab-Simulink) but not by a lab experiment. This was because there are major limitations in scaling down high-power equipment to low-power devices in a lab environment. Nonlinear devices, such as thyristors, cannot be scaled down to different voltages. Also, high power inductors have the $q$-factor, meaning the ratio of the resistance to reactance, of the order of 100 or above. Low power inductors, used in labs, have the $q$-factor at the level of only 10 or even below. Consequently, a lab model can have properties substantially different than the original system and the adaptive compensator.

\section{A Rationale of the Approach}

The power factor of electrical loads degrades not only because of the reactive power but also because of the load imbalance. The supply voltage and current harmonics could also contribute to its degradation.

The first balancing compensator, but only for a sinusoidal supply voltage, was developed by Steinmetz, and this compensator is known now as a Steinmetz circuit $[1,12]$. Some other approaches to load balancing were also suggested by Mayer and Kropik [13]. To this moment, the major obstacles in the development of the reactive compensators were theoretical, mainly caused by a controversy as to powers in three-phase systems with unbalanced loads and nonsinusoidal supply voltage [14-17, 24]. Eventually, the fundamentals for load balancing at nonsinusoidal voltage were developed in the frame of the current orthogonal components, later referred to as the CPC - based power theory [7, 17-18].

The load power, power factor, and load imbalance usually change in time, so the compensators should have the adaptive property. Adaptive compensators can be built as reactive compensators, with thyristors as the controlling devices, or as switching compensators (SCs), known commonly as active power filters, which use power transistors for the compensator control. When the load power is in the range of hundreds MVA, as this is common for large manufacturing plants, in particular, metallurgic ones, the power of SCs could not be sufficient for their compensation [25]. Reactive compensation could be the only option.

There are methods of reactive compensator design based on optimization procedures but a lot of computation could be needed for such procedures. Having in mind adaptive, in real-time compensation, the method of compensator's parameters calculation from direct mathematical expressions, rather than from optimization procedures seems to be more appropriate.

Just such a method provides the CPC - based power theory, which interprets power-related phenomena in electrical systems but also creates fundamentals for their compensation [17, 18]. The CPC-based method of reactive compensation specifies the theoretical limits of such compensation and the LC parameters of a fixed-parameters compensator. It can be next converted to an adaptive device.

As was suggested by Steeper and Stratford, a thyristor switched inductor (TSI) with a shunt capacitor, shown in Figure 1, can be used for that [19-20].

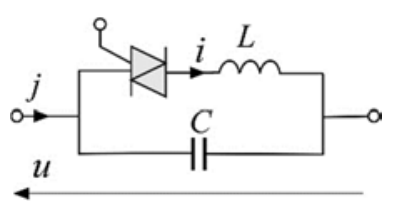

Figure 1. A thyristor-switched inductor with a shunt capacitor.

The TSI is a source of current harmonics of the level dependent on the thyristor firing angle. The harmonic of the third order is usually the dominating one. The adaptive compensators are built mainly as compensators of only the 
reactive power, however, and consequently, they are symmetrical devices, with all thyristors switched at the same angle. Due to this symmetry, the third-order current harmonics do not leave the compensator to the compensated system. When the compensator is used as a balancing device, then the firing angles are different and the third-order harmonics in the compensator branches do not cancel mutually in the compensator but disturb the compensating system. Moreover, in the presence of harmonics in the supply voltage, the resonance of the supply system inductance with the compensator capacitance $C$ can occur, causing substantial harmonic distortion in the compensated system. Countermeasures for that have to be provided. A TSI has to be integrated with resonant filters for that. Such filters should prevent, moreover, the system against the resonance of the compensator's equivalent capacitance with the supply system inductance.

Aggregates of single-phase computer-like loads as well as lightning, instrumentation equipment, and electrical transportation are the main cause of the load imbalance in manufacturing plants. Because a neutral conductor is needed for the supply of such loads, distribution systems in manufacturing plants are built as four-wire systems, shown in Figure 2.

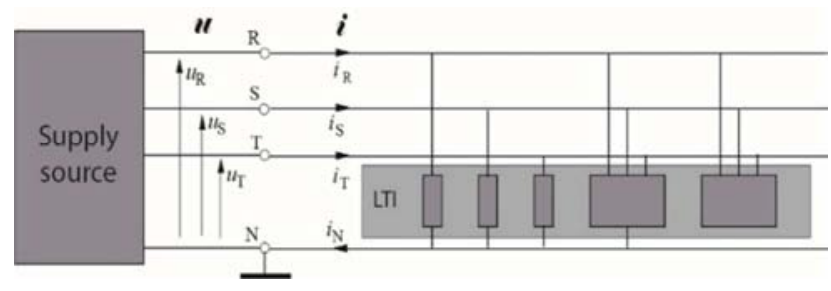

Figure 2. A structure of a three-phase, four-wire system.

Due to nonlinearity or periodic switching, the loads can generate current harmonics. Reactive compensators do not have any capability of compensating them, however. The load in Figure 2 is regarded therefore as a linear load.

Harmonic currents can be reduced, after the load for the fundamental frequency is compensated, by an additional switching compensator, known usually as an active power filter [21], of reduced power, because it does not have to compensate the harmful components of the supply currents' fundamental harmonic.

\section{Currents' Physical Components}

A distorted voltage in three-phase systems can be approximated by a sum of dominating harmonics of the order $n$ a set $N$, expressed $[7,9]$ as a three-phase vector:

$$
\boldsymbol{u}(t)=\sum_{n \in N} \boldsymbol{u}_{n}(t)=\sqrt{2} \operatorname{Re} \sum_{n \in N}\left[\begin{array}{c}
\boldsymbol{U}_{\mathrm{R} n} \\
\boldsymbol{U}_{\mathrm{S} n} \\
\boldsymbol{U}_{\mathrm{T} n}
\end{array}\right] e^{j n \omega_{1} t}=\sqrt{2} \operatorname{Re} \sum_{n \in N} \boldsymbol{U}_{n} e^{j n \omega_{1} t}
$$

where $U_{L n}$ is the complex rms (crms) value of the $n$-th order supply voltage harmonic at $L$ terminal, $L=\{\mathrm{R}, \mathrm{S} T\}$. Similarly, the load current can be approximated by the three-phase current vector

$$
\boldsymbol{i}(t)=\sum_{n \in N} \boldsymbol{i}_{n}(t)=\sqrt{2} \operatorname{Re} \sum_{n \in N}\left[\begin{array}{c}
\boldsymbol{I}_{\mathrm{R} n} \\
\boldsymbol{I}_{\mathrm{S} n} \\
\boldsymbol{I}_{\mathrm{T} n}
\end{array}\right] e^{j n \omega_{1} t}=\sqrt{2} \operatorname{Re} \sum_{n \in N} \boldsymbol{I}_{n} e^{j n \omega_{1} t}
$$

where $I_{L n}$ is the crms value of the $n$-th order load current harmonic at $L$ terminal. The load current can be decomposed, according to the Currents' Physical Components CPC) - based power theory, into six physical components [8]

$$
\boldsymbol{i}=\boldsymbol{i}_{\mathrm{a}}+\boldsymbol{i}_{\mathrm{s}}+\boldsymbol{i}_{\mathrm{r}}+\boldsymbol{i}_{\mathrm{u}}^{\mathrm{p}}+\boldsymbol{i}_{\mathrm{u}}^{\mathrm{n}}+\boldsymbol{i}_{\mathrm{u}}^{\mathrm{z}}
$$

In this decomposition

$$
\boldsymbol{i}_{\mathrm{a}}=\left[\begin{array}{c}
i_{\mathrm{Ra}} \\
i_{\mathrm{Sa}} \\
i_{\mathrm{Ta}}
\end{array}\right]=G_{\mathrm{e}} \boldsymbol{u}=\sqrt{2} \operatorname{Re} \sum_{n \in N} G_{\mathrm{e}} \boldsymbol{U}_{n} e^{j n \omega_{1} t}, \quad G_{\mathrm{e}}=\frac{P}{\|\boldsymbol{\mu}\|^{2}}
$$

is the active current. It is the current component needed to supply the load at voltage $\boldsymbol{u}$ with the active power $P$. The symbol $\|\boldsymbol{\mu}\|$ denotes the three-phase rms value of the supply voltage, defined [7] for a three-phase quantity $\boldsymbol{x}(t)$ as

$$
\|\boldsymbol{x}\|=\sqrt{\left\|x_{\mathrm{R}}\right\|^{2}+\left\|x_{\mathrm{S}}\right\|^{2}+\left\|x_{\mathrm{T}}\right\|^{2}} .
$$

The component $\boldsymbol{i}_{\mathrm{S}}$ in decomposition (3) is the scattered current. It is defined as

$$
\boldsymbol{i}_{\mathrm{S}}=\sqrt{2} \operatorname{Re} \sum_{n \in N}\left(G_{\mathrm{e} n}-G_{\mathrm{e}}\right) \boldsymbol{U}_{n} e^{j n \omega_{1} t}, \quad G_{\mathrm{e} n}=\frac{P_{n}}{\left\|\boldsymbol{u}_{n}\right\|^{2}}
$$

is the load equivalent conductance for the $n$-th order harmonic.

The symbol $P_{n}$ in formula (6) denotes the active power of the $n$-th order harmonic and $\left\|\boldsymbol{u}_{n}\right\|$ its three-phase rms value.

The component $\boldsymbol{i}_{\mathrm{r}}$ in decomposition (3) is the reactive current. It is defined as

$$
\boldsymbol{i}_{\mathrm{r}}=\sqrt{2} \operatorname{Re} \sum_{n \in N} j B_{\mathrm{e} n} \boldsymbol{U}_{n} e^{j n \omega_{1} t}, \quad B_{\mathrm{e} n}=-\frac{Q_{n}}{\left\|\boldsymbol{u}_{n}\right\|^{2}}
$$

The three components in decomposition (3) with upper indices $\mathrm{p}, \mathrm{n}$, and $\mathrm{z}$,

$$
\boldsymbol{i}_{\mathrm{u}}^{\mathrm{p}}+\boldsymbol{i}_{\mathrm{u}}^{\mathrm{n}}+\boldsymbol{i}_{\mathrm{u}}^{\mathrm{z}}=\boldsymbol{i}_{\mathrm{u}}
$$

stand for the positive, negative, and the zero sequence symmetrical components of the unbalanced current $\boldsymbol{i}_{\mathrm{u}}$ of the load. With symbols $\mathbf{1}^{\mathrm{p}}, \mathbf{1}^{\mathrm{n}}$, and $\mathbf{1}^{\mathrm{z}}$, explained in Figure 3

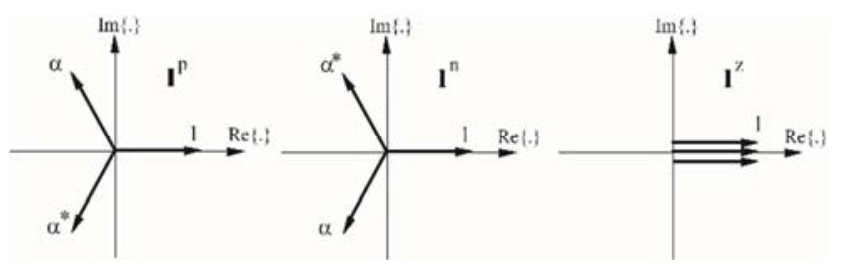

Figure 3. Unit symmetrical vectors.

the symmetrical components of the unbalanced current can be expressed as follows [8] 


$$
\begin{aligned}
& \boldsymbol{i}_{\mathrm{u}}^{\mathrm{p}}=\sum_{n \in N} \boldsymbol{i}_{\mathrm{u} n}^{\mathrm{p}}=\sqrt{2} \operatorname{Re} \sum_{n \in N} \boldsymbol{Y}_{\mathrm{u} n}^{\mathrm{p}} \mathbf{1}^{\mathrm{p}} \boldsymbol{U}_{\mathrm{R} n} e^{j n \omega_{1} t} \\
& \boldsymbol{i}_{\mathrm{u}}^{\mathrm{n}}=\sum_{n \in N} \boldsymbol{i}_{\mathrm{u} n}^{\mathrm{n}}=\sqrt{2} \operatorname{Re} \sum_{n \in N} \boldsymbol{Y}_{\mathrm{u} n}^{\mathrm{n}} \mathbf{1}^{\mathrm{n}} \boldsymbol{U}_{\mathrm{R} n} e^{j n \omega_{1} t} \\
& \boldsymbol{i}_{\mathrm{u}}^{\mathrm{z}}=\sum_{n \in N} \boldsymbol{i}_{\mathrm{u} n}^{\mathrm{z}}=\sqrt{2} \operatorname{Re} \sum_{n \in N} \boldsymbol{Y}_{\mathrm{u} n}^{\mathrm{z}} \mathbf{1}^{\mathrm{z}} \boldsymbol{U}_{\mathrm{R} n} e^{j n \omega_{1} t}
\end{aligned}
$$

Admittances $\boldsymbol{Y}_{\mathrm{u} n}^{\mathrm{p}}, \boldsymbol{Y}_{\mathrm{u} n}^{\mathrm{n}}$, and $\boldsymbol{Y}_{\mathrm{u} n}^{\mathrm{Z}}$ in last formulas are unba- lanced admittances of the positive, negative, and the zero sequence of the load. All these admittances can be calculated, if the line-to-neutral equivalent admittances for harmonic frequencies $Y_{\mathrm{R} n}, Y_{\mathrm{S} n}$, and $Y_{\mathrm{T} n}$, of the equivalent load, shown in Figure 4, are known. These admittances can be obtained by a measurement of the crms values of the voltage and current harmonics at the load terminals, namely

$$
\boldsymbol{Y}_{L n}=G_{L n}+j B_{L n}=\frac{\boldsymbol{I}_{L n}}{\boldsymbol{U}_{L n}}, \quad L=\mathrm{R}, \mathrm{S} \text { or T }
$$

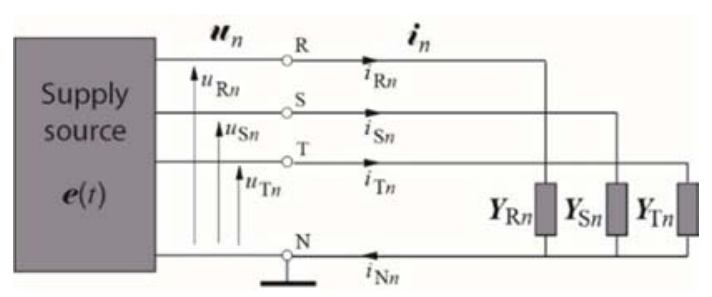

Figure 4. An equivalent circuit of the LTI load.

With these line-to-neutral equivalent admittances $Y_{\mathrm{R} n}, Y_{\mathrm{S} n}$, and $Y_{\mathrm{T} n}$, for harmonic frequencies, the unbalanced admittances of particular symmetrical sequences can be obtained, namely

$$
\boldsymbol{Y}_{\mathrm{u} n}^{\mathrm{p}}=\frac{1}{3}\left[\left(\boldsymbol{Y}_{\mathrm{R} n}+\alpha \beta \boldsymbol{Y}_{\mathrm{S} n}+\alpha^{*} \beta^{*} \boldsymbol{Y}_{\mathrm{T} n}\right)-\boldsymbol{Y}_{\mathrm{e} n}\left(1+\alpha \beta+\alpha^{*} \beta^{*}\right)\right]
$$

where

$$
\begin{gathered}
\boldsymbol{Y}_{\mathrm{e} n}=G_{\mathrm{e} n}+j B_{\mathrm{e} n}=\frac{1}{3}\left(\boldsymbol{Y}_{\mathrm{R} n}+\boldsymbol{Y}_{\mathrm{S} n}+\boldsymbol{Y}_{\mathrm{T} n}\right) \\
\beta \stackrel{\mathrm{df}}{=}\left(\alpha^{*}\right)^{n}=\left\{\begin{array}{ccc}
1, & \text { for } & n=3 k \\
\alpha^{*}, & \text { for } & n=3 k+1, \\
\alpha, & \text { for } & n=3 k-1
\end{array}\right.
\end{gathered}
$$

for the negative sequence harmonics

$$
\boldsymbol{Y}_{\mathrm{u} n}^{\mathrm{n}}=\frac{1}{3}\left[\left(\boldsymbol{Y}_{\mathrm{R} n}+\alpha^{*} \beta \boldsymbol{Y}_{\mathrm{S} n}+\alpha \beta^{*} \boldsymbol{Y}_{\mathrm{T} n}\right)-\boldsymbol{Y}_{\mathrm{e} n}\left(1+\alpha^{*} \beta+\alpha \beta^{*}\right)\right]
$$

and for the zero sequence harmonics

$$
\boldsymbol{Y}_{\mathrm{u} n}^{z}=\frac{1}{3}\left[\left(\boldsymbol{Y}_{\mathrm{R} n}+\beta \boldsymbol{Y}_{\mathrm{S} n}+\beta^{*} \boldsymbol{Y}_{\mathrm{T} n}\right)-\boldsymbol{Y}_{\mathrm{e} n}\left(1+\beta+\beta^{*}\right)\right]
$$

The Currents' Physical Components in decomposition (3), are mutually orthogonal [9]. Thus, they contribute to the three-phase rms value of the supply current independently of each other, namely

$$
\|\boldsymbol{i}\|^{2}=\left\|\dot{\boldsymbol{i}}_{\mathrm{a}}\right\|^{2}+\left\|\boldsymbol{i}_{\mathrm{s}}\right\|^{2}+\left\|\boldsymbol{i}_{\mathrm{r}}\right\|^{2}+\left\|\dot{\boldsymbol{i}}_{\mathrm{u}}^{\mathrm{p}}\right\|^{2}+\left\|\boldsymbol{i}_{\mathrm{u}}^{\mathrm{n}}\right\|^{2}+\left\|\boldsymbol{i}_{\mathrm{u}}^{\mathrm{z}}\right\|^{2}
$$

Only the active current $\boldsymbol{i}_{\mathrm{a}}$ is necessary for the permanent transmission of energy to the load. The remaining ones increase the supply current three-phase rms value. They reduce the load power factor thus, they are harmful.

\section{Reactive Compensation}

The power factor can be improved by the reduction of the harmful components of the supply current. the scattered current $\boldsymbol{i}_{\mathrm{s}}$ cannot be compensated by a shunt reactive compensator [7]. Such a compensator, shown in Figure 5, has to be composed of two sub-compensators with $\mathrm{Y}$ and $\Delta$ structures, which can reduce only the reactive and unbalanced currents [10]. The compensator in Figure 5 is specified by susceptances of its branches for the $n^{\text {th }}$-order harmonic.

As assumed in this article, the load is linear, similarly to the compensator, so that the whole system satisfies the Superposition Principle, so that, in the presence of the supply voltage distortion, it can be analyzed harmonic-by-harmonic.

Compensation of the zero-sequence symmetrical component of the unbalanced current $\boldsymbol{i}_{\mathrm{u}}^{\mathrm{z}}$ is possible only when the compensator provides a pass for such a current. Thus, it has to be configured in Y. It compensates this current entirely on the condition that suceptance of the Y sub-compensator branches for harmonic frequencies, $T_{\mathrm{R} n}, T_{\mathrm{S} n}$, and $T_{\mathrm{T} n}$, satisfy for each $n$ from the set $N$, the equation

$$
\frac{1}{3} j\left(T_{\mathrm{R} n}+\alpha^{*} T_{\mathrm{S} n}+\alpha T_{\mathrm{T} n}\right)+\boldsymbol{Y}_{\mathrm{u} n}^{\mathrm{z}}=0
$$

This equation has to be satisfied for the real and the imaginary parts of the complex coefficients of this equation thus, it has an infinite number of solutions with regard to three unknown susceptances of the compensator branches. It has only one solution if this $\mathrm{Y}$ sub-compensator has to compensate entirely also the reactive current $\boldsymbol{i}_{\mathrm{r}}$, so that its susceptances have to satisfy the additional equation

$$
\frac{1}{3}\left(T_{\mathrm{R} n}+T_{\mathrm{S} n}+T_{\mathrm{T} n}\right)+B_{\mathrm{e} n}=0 .
$$

Equations (19) and (20) result in the susceptances for harmo- nic frequencies of the Y sub-compensator branches, namely

$$
\begin{aligned}
& T_{\mathrm{R} n}=-2 \operatorname{Im} \boldsymbol{Y}_{\mathrm{u} n}^{\mathrm{z}}-B_{\mathrm{e} n} \\
& T_{\mathrm{S} n}=-\sqrt{3} \operatorname{Re} \boldsymbol{Y}_{\mathrm{u} n}^{\mathrm{z}}+\operatorname{Im} \boldsymbol{Y}_{\mathrm{u} n}^{\mathrm{z}}-B_{\mathrm{e} n} . \\
& T_{\mathrm{T} n}=\sqrt{3} \operatorname{Re} \boldsymbol{Y}_{\mathrm{u} n}^{\mathrm{z}}+\operatorname{Im} \boldsymbol{Y}_{\mathrm{u} n}^{\mathrm{z}}-B_{\mathrm{e} n}
\end{aligned}
$$

Such a compensator eliminates components $\boldsymbol{i}_{\mathrm{u}}^{\mathrm{Z}}$ and $\boldsymbol{i}_{\mathrm{r}}$ from the supply current entirely, while the remaining ones are not changed. A second sub-compensator of $\Delta$ structure can be used for their reduction. Since the scattered current $\boldsymbol{i}_{\mathrm{s}}$ cannot be compensated by a shunt reactive compensator, only two 
symmetrical components of the unbalanced current, namely $\boldsymbol{i}_{\mathrm{u}}^{\mathrm{p}}$ and $\boldsymbol{i}_{\mathrm{u}}^{\mathrm{n}}$ remain for compensation.

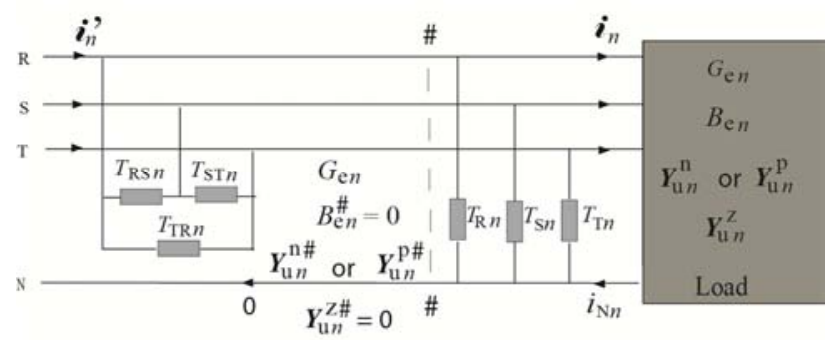

Figure 5. Load with a reactive compensator for the $n^{\text {th }}$ - order harmonic of the positive or negative sequence.

The sub-compensator of $\Delta$-structure, connected at the supply terminals, as shown in Figure 5, has to compensate the load with parameters modified by the sub-compensator of the Y structure.

The partially compensated load, as seen from the crosssection \#-\#, has the equivalent susceptance for harmonic of the order $n$ from the set $N, B_{\mathrm{e} n}^{\#}$ and the unbalanced admittance of the zero-sequence $\boldsymbol{Y}_{\mathrm{u} n}^{\mathrm{Z}}$ reduced to zero, while two remaining ones are changed to $\boldsymbol{Y}_{\mathrm{u} n}^{\mathrm{p} \#}$ and $\boldsymbol{Y}_{\mathrm{u} n}^{\mathrm{n} \#}$, respectively.

A sub-compensator of the $\Delta$ structure compensates the unbalanced current of the negative sequence [10] on the condition that

$$
j\left(T_{\mathrm{ST} n}+\alpha T_{\mathrm{TR} n}+\alpha^{*} T_{\mathrm{RS} n}\right)+\boldsymbol{Y}_{\mathrm{u} n}^{\mathrm{n} \#}=0 .
$$

and the positive sequence on the condition that

$$
j\left(T_{\mathrm{ST} n}+\alpha^{*} T_{\mathrm{TR} n}+\alpha T_{\mathrm{RS} n}\right)+\boldsymbol{Y}_{\mathrm{u} n}^{\mathrm{p} \#}=0 .
$$

Because of complex coefficients, equations (22) and (23) stand for four equations. They cannot be satisfied for three unknown branch susceptances. Therefore, one of the equations (22) and (23) has to be abandoned, which means that only one symmetrical component of the unbalanced current, $\boldsymbol{i}_{\mathrm{u}}^{\mathrm{p}}$ or $\boldsymbol{i}_{\mathrm{u}}^{\mathrm{n}}$, can be compensated.

The positive sequence component of the unbalanced current $\boldsymbol{i}_{\mathrm{u}}^{\mathrm{p}}$ occurs only [9] if the supply voltage has harmonics of the negative sequence, $n=2,5 \ldots n=(3 k-1)$, or the zero sequence, $n=3,6 \ldots n=3 k$. The fundamental harmonic dominates usually in the negative sequence component $\boldsymbol{i}_{\mathrm{u}}^{\mathrm{n}}$. Therefore, the component $\boldsymbol{i}_{\mathrm{u}}^{\mathrm{p}}$ is usually much smaller than the component $\boldsymbol{i}_{\mathrm{u}}^{\mathrm{n}}$ and consequently, the last one can be left uncompensated. It means that the branch susceptances of the subcompensator of $\Delta$ structure should satisfy only equation (22). Since this sub-compensator should not load the supply source with the reactive current, these susceptances have to satisfy moreover, for each $n$ from the set $N$, the condition

$$
T_{\mathrm{ST} n}+T_{\mathrm{TR} n}+T_{\mathrm{RS} n}=0 .
$$

As to equation (22), we have to remember that the subcompensator of Y structure modifies the unbalanced admittance of the negative sequence as seen from the supply source [10]. It is equal to

$$
\boldsymbol{Y}_{\mathrm{u} n}^{\mathrm{n} \#}=\boldsymbol{Y}_{\mathrm{u} n}^{\mathrm{Z}^{*}}+\boldsymbol{Y}_{\mathrm{u} n}^{\mathrm{n}}
$$

With this admittance, equations (22) and (24) results in the sub-compensator susceptances

$$
\begin{aligned}
& T_{\mathrm{RS} n}=\frac{1}{3}\left(\sqrt{3} \operatorname{Re} \boldsymbol{Y}_{\mathrm{u} n}^{\mathrm{n} \#}-\operatorname{Im} \boldsymbol{Y}_{\mathrm{u} n}^{\mathrm{n} \#}\right) \\
& T_{\mathrm{ST} n}=\frac{1}{3}\left(2 \operatorname{Im} \boldsymbol{Y}_{\mathrm{u} n}^{\mathrm{n} \#}\right) \\
& T_{\mathrm{TR} n}=\frac{1}{3}\left(-\sqrt{3} \operatorname{Re} \boldsymbol{Y}_{\mathrm{u} n}^{\mathrm{n} \#}-\operatorname{Im} \boldsymbol{Y}_{\mathrm{u} n}^{\mathrm{n} \#}\right)
\end{aligned}
$$

\section{Compensator Complexity Reduction}

Formulas (21) and (26) provide susceptances of all branches of a reactive compensator for harmonic frequencies needed for the entire compensation of the reactive as well as the zero and the negative symmetrical components of the unbalanced current. The structure and the $L C$ parameters of such branches can be found using well-developed methods of reactance one-ports synthesis [22]. Unfortunately, with increasing number of supply voltage harmonics, the number of inductors and capacitors needed for the compensator construction increases. Approximately, one extra inductor and one extra capacitor per branch of the compensator are needed for each extra voltage harmonic. For example, if $N=\{1,3,5,7\}$, then at least 40 reactance elements might be needed for the compensator construction. It would be too complex and consequently, too expensive to have a technical value.

The number of elements needed for the compensator construction can be reduced if the requirement of the entire compensation of the supply current components $\boldsymbol{i}_{\mathrm{r}}, \boldsymbol{i}_{\mathrm{u}}^{\mathrm{Z}}$, and $\boldsymbol{i}_{\mathrm{u}}^{\mathrm{p}}$, is abandoned for the only reduction of their three-phase rms value.

The most simple compensator has only one reactive element, capacitor or inductor, by a branch. Since capacitors in the compensator can result in a series resonance with the supply source inductance, purely capacitive branches are not acceptable. Therefore, the reduced complexity compensator cannot have branches other than those, shown in Figure 6.

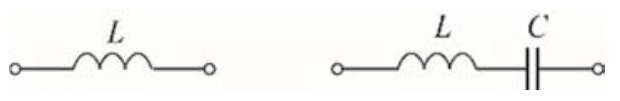

Figure 6. Acceptable branches of a reduced complexity compensator

The branch susceptances of the reduced complexity compensator are denoted by $D_{n}$, to distinguish them from the those calculated from formulas (21) and (26). They have for harmonic frequencies the values

$$
D_{n}=-\frac{1}{n \omega_{1} L} \quad \text { or } \quad D_{n}=\frac{n \omega_{1} C}{1-n^{2} \omega_{1}^{2} L C} .
$$

A compensator of the reduced complexity minimizes the 
supply current three-phase rms value on the condition, that the suceptance $D_{k n}$ of each branch $k$ is selected such that the following expression is minimized [10]

$$
\sum_{n \in N}\left(T_{k n}-D_{k n}\right)^{2} U_{k n}^{2}=\sum_{n \in N} A_{k n}^{2}=\mathrm{Min} .
$$

where susceptances $T_{k n}$ of these branches are given by formulae (21) and (26), respectively.

Selection of the specific branch, meaning one of the two shown in Figure 6, can be based on the sign of the calculated susceptance for the fundamental frequency, $T_{k 1}$. It is because the rms value of the supply voltage fundamental harmonic $U_{k 1}$ is usually much higher than this value for other harmonics. Consequently, the term

$$
A_{k 1}=\left(T_{k 1}-D_{k 1}\right)^{2} U_{k 1}^{2}
$$

in formula (28) is much higher than such terms for other harmonics. To have the value of $A_{k 1}$ as close to zero as possible, branch $k$ should be selected in such a way that its susceptance $D_{k 1}$ has the same sign as the susceptance $T_{k 1}$. Thus, when $T_{k 1}$ is negative, then a purely inductive branch should be chosen. Its inductance should minimize the term

$$
\sum_{n \in N}\left(T_{k n}+\frac{1}{n \omega L_{k}}\right)^{2} U_{k n}^{2}=\mathrm{Min}
$$

the optimum value of this inductance is

$$
L_{k, \mathrm{opt}}=-\frac{1}{\omega_{1}} \frac{\sum_{n \in N} \frac{1}{n^{2}} U_{k n}^{2}}{\sum_{n \in N} T_{k n} \frac{1}{n} U_{k n}^{2}} .
$$

When $T_{k 1}$ is positive, then $L C$ branch should be chosen such that its $L C$ parameters should minimize the term

$$
\sum_{n \in N}\left(T_{k n}-\frac{n \omega_{1} C_{k}}{1-n^{2} \omega_{1}^{2} L_{k} C_{k}}\right)^{2} U_{k n}^{2}=\text { Min. }
$$

The left side of this expression does not have minimum for finite values of the inductance $L_{k}$, however. Thus, any value can be chosen. The product $L_{k} C_{k}$ specifies the approximate value of the frequency of the branch resonance. Therefore, inductance $L_{k}$ should be selected such that this resonance will not occur for harmonic frequencies. It can be done, however, only in an iterative process, because the inductance $L_{k}$ affects the capacitance $C_{k}$. Calculation of this capacitance is also possible only by an iterative process because expression (32) cannot be rearranged into an explicit formula with regard to this capacitance. It can be calculated as a limit of a sequence of capacitances obtained iteratively, namely

$$
C_{k, s+1}=\frac{\sum_{n \in N} \frac{T_{k n} n U_{k n}^{2}}{1-n^{2} \omega_{1}^{2} L_{k} C_{k, s}}}{\omega_{1} \sum_{n \in N} \frac{n^{2} U_{k n}^{2}}{\left(1-n^{2} \omega_{1}^{2} L_{k} C_{k, s}\right)^{2}}}
$$

The method of synthesis of a compensator with reduced complexity was illustrated for the load shown in Figure 7, supplied with a symmetrical voltage of the fundamental harmonic rms value $U_{1}=240 \mathrm{~V}$ [10]. It was assumed that the supply voltage was distorted by the $3^{\text {rd }}, 5^{\text {th }}$, and $7^{\text {th }}$ order harmonics of relative rms value $U_{3}=2 \% U_{1}, U_{5}=3 \% U_{1}$, and $U_{7}=1.5 \% U_{1}$. There are also shown in Figure 7 the three-phase rms values of the load current physical components

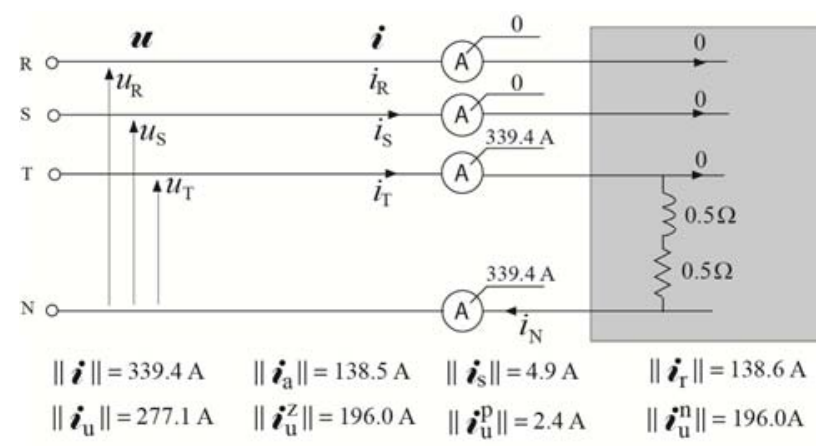

Figure 7. An example of an unbalanced load and results of its analysis.

The parameters of the reduced complexity compensator were calculated for the fundamental frequency normalized to $\omega_{1}=1 \mathrm{rad} / \mathrm{s}$, assuming that the resonant frequency of LC branches is $2.5 \mathrm{rad} / \mathrm{s}[10]$.

Table 1. LC parameters of a reduced complexity compensator.

\begin{tabular}{llllllll}
\hline & Line: & $\mathrm{R}$ & $\mathrm{S}$ & $\mathrm{T}$ & $\mathrm{RS}$ & $\mathrm{ST}$ & $\mathrm{TR}$ \\
\hline $\mathrm{L}$ & $\mathrm{mH}$ & 1730 & 770 & 444 & 0 & 2600 & 1155 \\
$\mathrm{C}$ & $\mathrm{mF}$ & 0 & 399 & 691 & 0 & 0 & 266 \\
\hline
\end{tabular}

The results of compensation are shown in Figure 8. The power factor is improved by the compensator of the reduced complexity from $\lambda=0.408$ to $\lambda=0.994$.

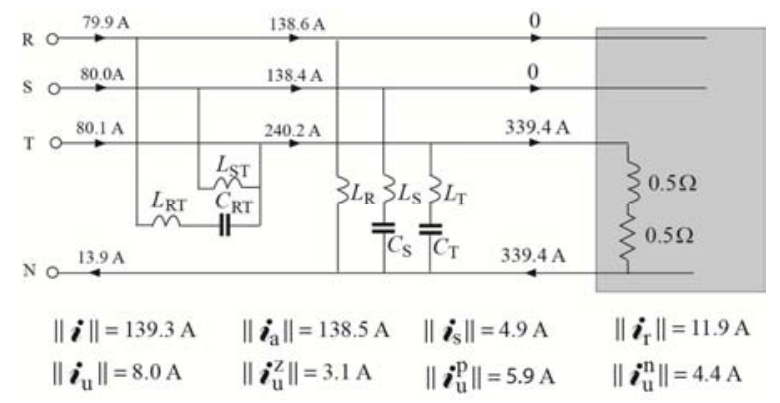

Figure 8. Results of compensation with a compensator of reduced complexity.

\section{Adaptive Compensator}

The compensator as developed above improves the power factor $\lambda$ of the supply source when its load has fixed parameters. When these parameters are not constant, such a compensator is losing effectiveness. An adaptive compensator is needed instead.

A reactive compensator has adaptive properties if it can be adjusted to changes in the load power of individual lines. This can be done by switches or by using reactive elements with controllable parameters. Saturation of the ferromagnetic core 
of an inductor enables a change of its inductance. A thyristorswitched inductor, shown in Figure 9a, is another and commonly used device of this category [19].
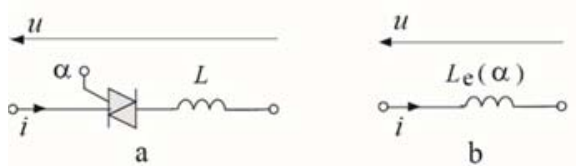

Figure 9. A thyristor switched inductor (a) and its equivalent inductance (b).

When it is connected in parallel with a capacitor, it serves as an adaptive compensator of the reactive power in systems with a sinusoidal supply voltage [19-20].

When the supply voltage is nonsinusoidal and the current harmonics generated by thyristors have to be reduced, the TSI is connected with a few reactive elements to shape the frequency properties of the compensator branches. Properties of such branches were studied by Czarnecki and Hsu [23]. Such branches, or reactive one-ports, will be referred to as thyristor-controlled susceptance (TCS) branches.

The current of a thyristor-switched inductor changes as shown in Figure 10. Symbol $i_{0}$ denotes current at $\alpha=0$.

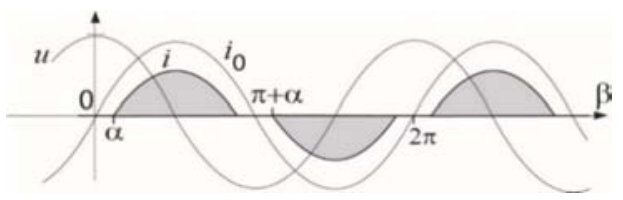

Figure 10. The voltage and currents waveform of TSI.

The equivalent admittance of the TSI for the fundamental frequency at a sinusoidal voltage at its terminal, expressed as a function of the firing angle $\alpha$, is equal to

$$
\boldsymbol{Y}_{1}=\frac{\boldsymbol{I}_{1}}{\boldsymbol{U}_{1}}=j B_{1}=\left(1-\frac{2 \alpha+\sin 2 \alpha}{\pi}\right) \frac{1}{j \omega_{1} L}=\frac{1}{j \omega_{1} L_{\mathrm{e}}(\alpha)}
$$

The current of thyristors is distorted from a sinusoidal waveform, so that TCS branches generate current harmonics. Usually, the $3^{\text {rd }}$ order harmonic is the dominating one. When a compensator is used only for the reactive current compensation, it is built as a symmetrical device, usually in $\Delta$ structure. Each branch of such a compensator generates the $3^{\text {rd }}$ order current harmonic of the same value and phase and consequently, it does not leave the $\Delta$ loop. This is no longer true in balancing compensators, which are unbalanced devices. They inject the 3rd order current harmonic into the compensated system, causing distortion. A parallel resonance of the TCS branch equivalent capacitance with the supply source inductance can occur as well.

The $3^{\text {rd }}$ order current harmonic leaving a TCS can be reduced by an $L C$ filter connected as shown in Figure 11, and tuned to the frequency of that harmonic.

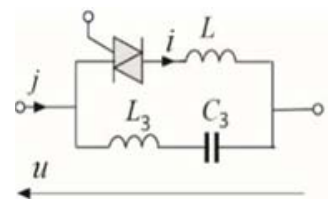

Figure 11. A TCS branch with a filter of the $3^{\text {rd }}$ order harmonic.

\section{Reduction Sensitivity to the Voltage Harmonics}

The filter reduces that harmonic, unfortunately, it creates a short circuit path for the $3^{\text {rd }}$ order harmonic in the supply voltage. To avoid it, an inductor denoted as $L_{0}$, can be added to the TCS branch as shown in Figure 12. It increases the impedance of the compensator as seen from the supply terminals for the $3^{\text {rd }}$ voltage harmonic. It increases moreover this impedance for frequencies above the frequency of the $3^{\text {rd }}$ order harmonic, where $L_{3} C_{3}$ branch has an inductive impedance.

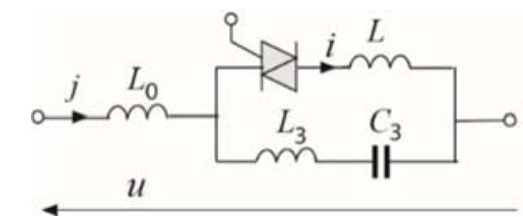

Figure 12. A TSC branch with a series inductor $L_{0}$.

The TCS branch is specified by four parameters, $L, L_{0}, L_{3}$, and $C_{3}$. There are only three conditions the TCS branch has to satisfy: the range of susceptance change, $T_{\min }, T_{\max }$, and resonant frequency of the $L_{3} C_{3}$ branch. Thus, one parameter can be selected at a designer's discretion [11].

After not being satisfied with harmonic distortion caused by the compensator, the authors concluded that there is one more phenomenon in the adaptive compensator that should be taken into account: namely, the voltage resonance of the whole TCS branch, at which its impedance approaches zero.

When the thyristor is in ON state, i.e., at firing angle $\alpha=0$, then the susceptance $T(\omega)$ of such a branch changes with the frequency as shown in Figure 13.

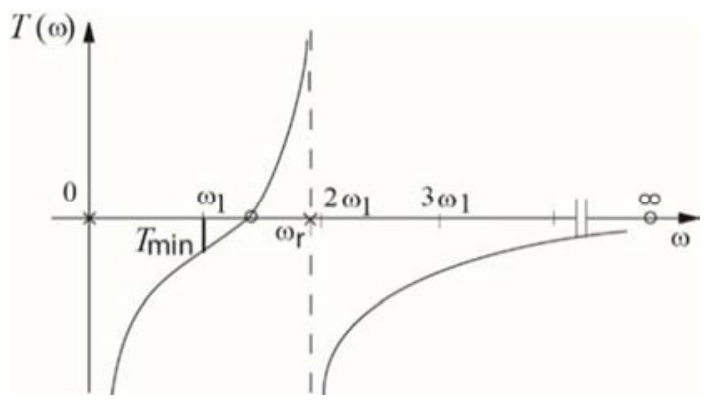

Figure 13. Change of the TCS branch in the thyristor ON state.

Its susceptance $T$ for the fundamental harmonic has the minimum value, equal to

$$
T_{\min }=\frac{9 \omega_{1}^{2} L C_{3}-8}{8 \omega_{1}\left(L_{0}+L\right)-9 \omega_{1}^{3} L L_{0} C_{3}} .
$$

When the thyristor is in OFF state, i.e., at $\alpha=90^{\circ}$, then the susceptance $T(\omega)$ of such a branch changes with frequency as shown in Figure 14. Its susceptance for the fundamental harmonic has the maximum value equal to

$$
T_{\max }=\frac{9 \omega_{1} C_{3}}{8-9 \omega_{1}^{2} L_{0} C_{3}} .
$$




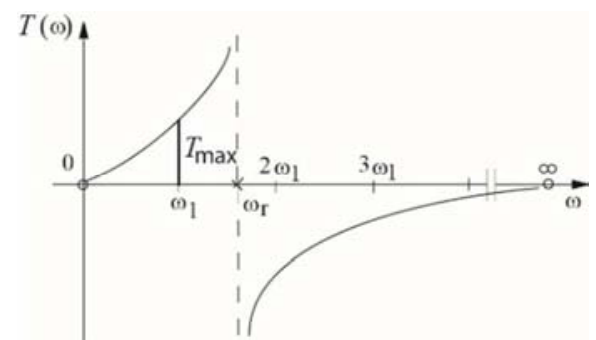

Figure 14. Change of the TCS branch in the thyristor OFF state.

At some frequency, denoted by $\omega_{\mathrm{r}}$, a voltage resonance of the whole TSC branch occurs. Its susceptance approaches infinity. Since the equivalent inductance of the TSI branch changes with the firing angle, the frequency $\omega_{\mathrm{r}}$ changes as well. Its maximum value is in the thyristor $\mathrm{ON}$ state. Its relative value, referenced to the fundamental frequency, equal to

$$
\frac{\omega_{\mathrm{r}}}{\omega_{1}}=\Omega=\sqrt{8} \sqrt{\frac{L_{3}\left(L+L_{0}\right)}{L L_{0}}} .
$$

To avoid resonance at the $2^{\text {nd }}$ order harmonic, which can be present in the supply voltage, the parameters of the TCS branch should be select in such a way that the relative resonance frequency (37) is below 2 .

The conditions (35), (36), (37), and the resonance frequency of the $L_{3} C_{3}$ branch, can be rearranged with regard to one of four parameters of the LSC branch. If inductance $L_{0}$ is such a parameter, then it has to satisfy the equation

$$
a_{3} L_{0}^{3}+a_{2} L_{0}^{2}+a_{1} L_{0}+a_{0}=0
$$

To compact symbols, let us denote $T_{\min }=T_{\mathrm{a}}, T_{\max }=T_{\mathrm{b}}$. With such symbols and the frequency $\omega_{1}$ normalized to 1 $\mathrm{rad} / \mathrm{s}$, coefficients of eqn. (42) are

$$
\begin{gathered}
a_{0}=9-\Omega^{2} \\
a_{1}=\left(27-11 \Omega^{2}\right) T_{\mathrm{b}} \\
a_{2}=\left[2 T_{\mathrm{b}}\left(9-5 \Omega^{2}\right)+9 T_{\mathrm{a}}\left(1-\Omega^{2}\right)\right] T_{\mathrm{b}} \\
a_{3}=9\left(1-\Omega^{2}\right) T_{\mathrm{a}} T_{\mathrm{b}}^{2}
\end{gathered}
$$

When eqn. (42) is solved, the parameters of the TCS branch can be expressed in terms of the inductance $L_{0}$ as follows

$$
\begin{gathered}
L_{3}=\left(L_{0}+1 / T_{\mathrm{b}}\right) / 8 \\
C_{3}=1 /\left(9 L_{3}\right) \\
L=\left[T_{\mathrm{a}} T_{\mathrm{b}} L_{0}^{2}+\left(T_{\mathrm{a}}+T_{\mathrm{b}}\right) L_{0}+1\right] /\left[\left(T_{\mathrm{b}}-T_{\mathrm{a}}\right)\right] .
\end{gathered}
$$

The needed range of the change of the branch's susceptance $T$ depends, of course, on the load: its reactive power and possible level of imbalance. This relatively complex issue is, however, beyond the scope of this paper, which is to only demonstrate that adaptive balancing in four-wire systems in the presence of the supply voltage distortion is possible. Therefore, the circuit used in the numerical illustration before will be used again to illustrate an adaptive balancing. The adaptive compensator will be designed at the assumption that the supply voltage is identical as before, while the individual supply lines are loaded randomly but no more than to the degree as line $\mathrm{T}$ load in Figure 7.

The needed minimum and maximum values of the susceptance, $T_{\min }, T_{\max }$, of TCS branches of sub-compensators can be found having optimized $L C$ values of the fixed-parameters compensator, previously calculated and compiled in Table 1. Thus, for the Y sub-compensator

$$
\begin{aligned}
& T_{\min }=-\frac{1}{\omega_{1} L_{\mathrm{R}}}=-\frac{1}{1.730}=-0.578 \mathrm{~S} \\
& T_{\max }=\frac{1}{\frac{1}{\omega_{1} C_{\mathrm{T}}}-\omega_{1} L_{\mathrm{T}}}=\frac{1}{\frac{1}{0.691}-0.444}=0.997 \mathrm{~S}
\end{aligned}
$$

and for the $\Delta$ sub-compensator

$$
\begin{gathered}
T_{\min }=-\frac{1}{\omega_{1} L_{\mathrm{ST}}}=-\frac{1}{2.60}=-0.385 \mathrm{~S} \\
T_{\max }=\frac{1}{\frac{1}{\omega_{1} C_{\mathrm{TR}}}-\omega_{1} L_{\mathrm{TR}}}=\frac{1}{\frac{1}{0.266}-1.155}=0.384 \mathrm{~S} .
\end{gathered}
$$

Assuming that $\Omega=1.9$, coefficients of eqn. (42), calculated with (43) - (44), have the values compiled in Table 2.

Table 2. Coefficients of the inductance $L_{0}$ equation.

\begin{tabular}{lllll}
\hline & $a_{0}$ & $a_{1}$ & $a_{2}$ & $a_{3}$ \\
\hline$\Delta$ & 5.39 & -4.88 & -0.004 & 1.34 \\
$\mathrm{Y}$ & 5.39 & -12.66 & 8.98 & 13.49 \\
\hline
\end{tabular}

Parameters of the $\Delta$ and Y sub-compensators TCS branches of the structure shown in Figure 12, calculated from formulas (47)-(49), are compiled in Table 3.

Table 3. Parameters of the compensators' TCS branches.

\begin{tabular}{lllll}
\hline & $\boldsymbol{L}_{\mathbf{0}}[\mathbf{H}]$ & $\boldsymbol{L}_{\mathbf{3}}[\mathbf{H}]$ & $\boldsymbol{C}_{\mathbf{3}}[\mathbf{F}]$ & $\boldsymbol{L}[\mathbf{H}]$ \\
\hline$\Delta$ & 1.100 & 0.463 & 0.240 & 1.065 \\
$\mathrm{Y}$ & 0.370 & 0.172 & 0.640 & 0.683 \\
\hline
\end{tabular}

The voltage and current at each TCS branch are in general, nonsinusoidal. Since the $3^{\text {rd }}$ order harmonic is usually the dominating one in the distorted current of the TSI branch, the $L_{3} C_{3}$ filter reduces the harmonic distortion of the TCS branch current $j$ substantially. Therefore, the TCS branch, as shown in Figure 12, can be approximated by an equivalent branch for the fundamental harmonic, as shown in Figure 15.

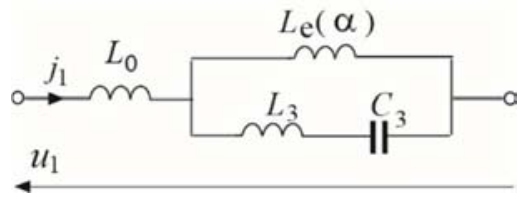

Figure 15. An equivalent TCS branch for the fundamental harmonic.

Its susceptance for the normalized fundamental frequency is 


$$
T=\frac{9 L_{\mathrm{e}}(\alpha) C_{3}-8}{8\left[L_{0}+L_{\mathrm{e}}(\alpha)\right]-9 L_{\mathrm{e}}(\alpha) L_{0} C_{3}}=T(\alpha) .
$$

This formula, for a given value of the firing angle $\alpha$, provides the branch susceptance $T$. It cannot be solved, however, with respect to the firing angle $\alpha$. A look-up table, which for angles in the range from $0^{\circ}$ to $90^{\circ}$ specifies the susceptance $T$ of TCS branches of both $\mathrm{Y}$ and $\Delta$ sub- compensators, is needed.

When the compensator has the structure and parameters as shown in Figure 8, then for the Y sub-compensator:

$$
\begin{aligned}
& T_{\mathrm{R}}=T_{\min }=-\frac{1}{\omega_{1} L_{\mathrm{R}}}=-0.578 \mathrm{~S} ; \quad \alpha_{\mathrm{R}}=0^{\circ} \\
& T_{\mathrm{S}}=\frac{1}{\frac{1}{\omega_{1} C_{\mathrm{S}}}-\omega_{1} L_{\mathrm{S}}}=0.491 \mathrm{~S} ; \quad \alpha_{\mathrm{S}}=47.3^{\circ} \\
& T_{\mathrm{T}}=T_{\max }=\frac{1}{\frac{1}{\omega_{1} C_{\mathrm{T}}}-\omega_{1} L_{\mathrm{T}}}=0.576 \mathrm{~S} ; \quad \alpha_{\mathrm{T}}=90^{\circ}
\end{aligned}
$$

and for the $\Delta$ sub-compensator

$$
\begin{array}{ll}
T_{\mathrm{RS}}=0 ; & \alpha_{\mathrm{RS}}=38^{\circ} \\
T_{\mathrm{ST}}=T_{\min }=-\frac{1}{\omega_{1} L_{\mathrm{ST}}}=-0.385 \mathrm{~S} ; & \alpha_{\mathrm{ST}}=0^{\circ} \\
T_{\mathrm{TR}}=T_{\max }=\frac{1}{\frac{1}{\omega_{1} C_{\mathrm{TR}}}-\omega_{1} L_{\mathrm{TR}}}=0.384 \mathrm{~S} ; & \alpha_{\mathrm{TR}}=90^{\circ} .
\end{array}
$$

The results of compensation are shown in Figure 16. These results confirm the possibility of an adaptive compensation of unbalanced linear loads supplied by a four-wire line in the presence of the supply voltage distortion. Similarly, as in the case of compensation by a fixed-parameters compensator of reduced complexity, some residual parts of the reactive and unbalanced currents remain.

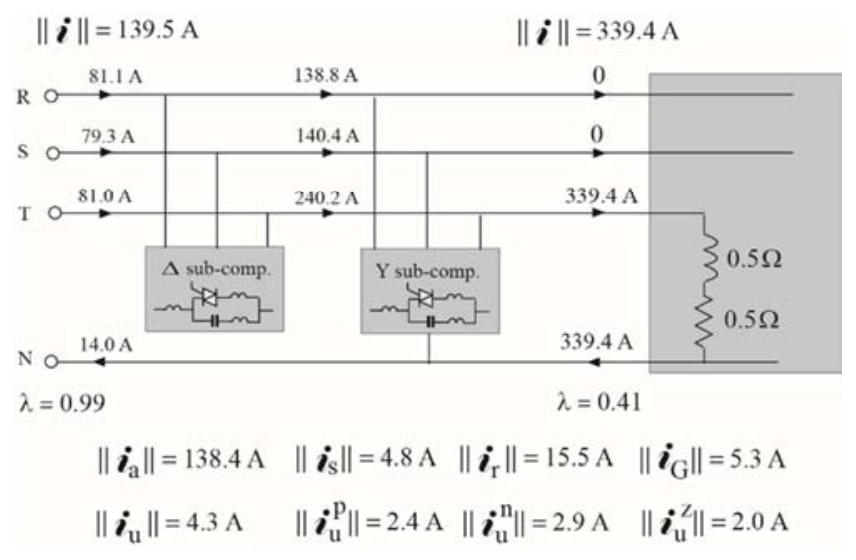

Figure 16. Results of adaptive compensation.

The waveform of the supply current in line R, after adaptive compensation, is shown in Figure 17. This current is in-phase with the supply voltage, but it is distorted. It is because the compensator does not compensate harmonics but some physical components of the supply current. All not compensa- ted components, even the active current, are distorted.

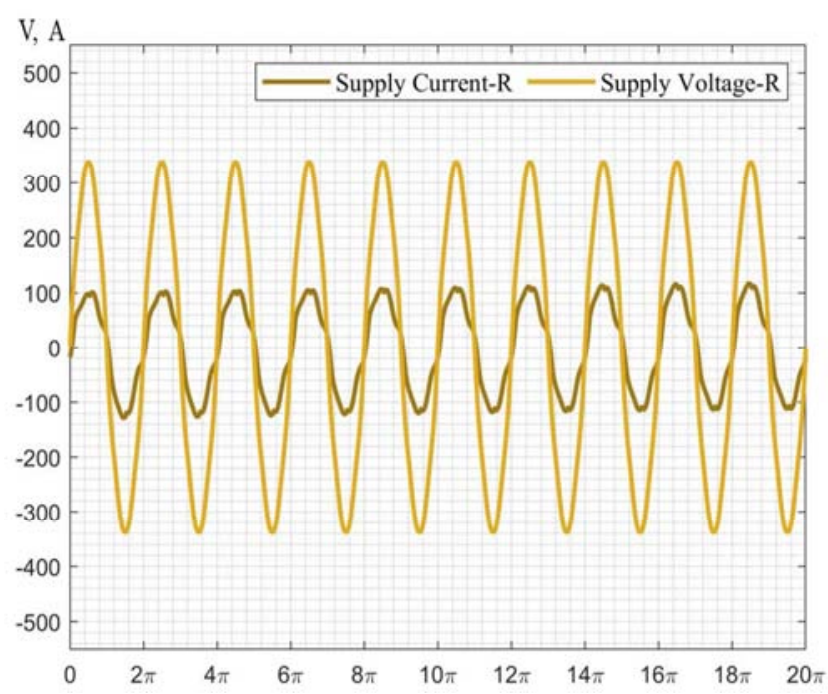

Figure 17. Voltage and current waveforms in supply line $R$.

The content of harmonics in the supply current is shown in Figure 18. It enables to compare this content when the compensator is designed based on an engineering intuition (blue) [11], with that based on the approach presented in this paper (red).

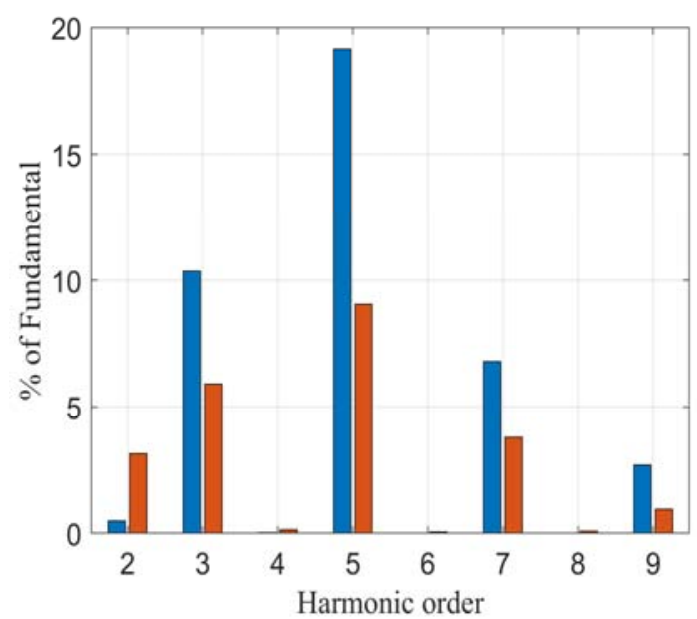

Figure 18. The contents of harmonics in the supply current of a compensator designed by an engineering intuition [11] (blue) and in this paper (red).

\section{Compensator-Generated Harmonics}

Distortion of the compensator current by thyristors means that apart from the supply voltage originated harmonics, also the compensator-originated harmonics can occur in the supply current, $\boldsymbol{i}_{\mathrm{S}}$.

Let us denote the supply current in the system with the compensator but with removed thyristor branches, by $\boldsymbol{i}_{\mathrm{S} 0}$. The difference

$$
\boldsymbol{i}_{\mathrm{S}}-\boldsymbol{i}_{\mathrm{S} 0}=\boldsymbol{i}_{\mathrm{G}}
$$

approximates the compensator-generated harmonic current $\boldsymbol{i}_{\mathrm{G}}$. The harmonics of the compensator-generated current $\boldsymbol{i}_{\mathrm{G}}$ 
are created by thyristors switching. The effect of the voltage harmonics upon thyristors' switching is negligible so that harmonics of the currents $\boldsymbol{i}_{\mathrm{S} 0}$ and $\boldsymbol{i}_{\mathrm{G}}$ are mutually random, thus these two currents are mutually orthogonal. Hence,

$$
\left\|\dot{\boldsymbol{i}}_{\mathrm{S}}\right\|^{2}=\left\|\boldsymbol{i}_{\mathrm{S} 0}\right\|^{2}+\left\|\boldsymbol{i}_{\mathrm{G}}\right\|^{2}
$$

When the line-to-neutral magnitude of admittances for harmonics of the compensator without thyristors are denoted by $Y$ with an apostrophe, then the three-phase rms value of such a compensator current can be expressed as

$$
\left\|\boldsymbol{i}_{\mathrm{S} 0}\right\|^{2}=\sum_{n \in N}\left\|\boldsymbol{i}_{\mathrm{S} 0 n}\right\|^{2}=\sum_{n \in N}\left[\left(Y_{\mathrm{R} n}^{\prime} U_{\mathrm{R} n}\right)^{2}+\left(Y_{\mathrm{S} n}^{\prime} U_{\mathrm{S} n}\right)^{2}+\left(Y_{\mathrm{R} n}^{\prime} U_{\mathrm{T} n}\right)^{2}\right]
$$

and hence, the three-phase rms value of the compensatorgenerated current is

$$
\left\|\boldsymbol{i}_{\mathrm{G}}\right\|=\sqrt{\left\|\boldsymbol{i}_{\mathrm{S}}\right\|^{2}-\left\|\boldsymbol{i}_{\mathrm{S} 0}\right\|^{2}}
$$

This value shown in Figure 16 was calculated just according to the above formula.

\section{Conclusions}

The paper shows that the presented method of synthesis of TCS branches of an adaptive compensator improves its performance in the presence of the supply voltage distortion. The adaptive compensator of unbalanced loads supplied with a nonsinusoidal voltage, effectively reduces the reactive and unbalanced currents, without any substantial distortion of the supply current. Nonetheless, this distortion still exists and it is not caused only by the thyristor switched inductors. This is because the active current, which is not the subject of compensation, reproduces the supply voltage distortion. Moreover, the scattered current is not affected by reactive compensators. Also, only two of three symmetrical components of the unbalanced current can be compensated by such a compensator. Despite that, the compensator is very effective in balancing even strongly unbalanced loads and in improving their power factor to almost unity value. Moreover, unlike switching compensators, its power is less confined.

\section{References}

[1] Ch. P. Steinmetz, Theory and calculation of electrical apparatur, McGraw-Hill Book Comp., New York, 1917.

[2] M. M. A. Aziz, at all, "LC compensator for power factor correction of non-linear loads", IEEE Trans. on Power Delivery, Vol. 19, No. 1, pp. 331-335, 2004.

[3] S.-J. Jeon, "Passive-component-based reactive power compensation in a non-sinusoidal multi-line system", Electrical Engineering, Vol. 102, pp. 1567-1577, 2020.

[4] D. Maiti, S. Mukhopadhyay, S. K. Biswas, "Three-phase thyristor controlled reactor using two sets of delta connected switches with low current harmonics", IET Power Electronics, DOI: 10.1049/iet-pel, 2019.
[5] S. Morello, T. J. Dionise, T. L. Mank, "Comprehensive analysis to specify a static var compensator for an arc furnace", IEEE Trans. on Ind. Appl., Vol. 51, No. 6, pp. 1153-1160, 2015.

[6] A. Luo, Z. Shuai, W. Zhu, Z. J. Shen, "Combined system for harmonic suppression and reactive power compensation", IEEE Trans. on Industrial Electronics, Vol. 56, No. 2, pp. 418-420, 2009.

[7] L. S. Czarnecki, "Orthogonal decomposition of the current in a three-phase nonlinear symmetrical circuit with nonsinusoidal voltage", IEEE Trans. on Instr. and Meas., Vol. M-37, No. 1, pp. 30-34, 1988.

[8] L. S. Czarnecki, "Reactive and unbalanced currents compensation in three-phase circuits under nonsinusoidal conditions", IEEE Trans. on Instr. and Meas., IM-38, No. 3, pp. 754-459, 1989.

[9] L. S. Czarnecki, P. H. Haley, "Power properties of four-wire systems with nonsinusoidal symmetrical voltage", IEEE Trans. on Power Delivery, Vol. 31, No. 2, pp. 513-521, 2016.

[10] L. S. Czarnecki, "CPC-based reactive balancing of linear loads in four-wire supply systems with nonsinusoidal voltage", Przeglad Elektrotechniczny, R. 95, Nr. 95, pp. 1-8, 2019.

[11] L. S. Czarnecki, M. Almousa, "Adaptive balancing of threephase loads at four-wire supply with reactive compensators and nonsinusoidal voltage, "2020 IEEE Texas Power and Energy Conf. (TPEC), A\&M University, USA, pp. 1-6, DOI: 10.1109/TPEC48276.2020.9042572, 2020.

[12] O. Jordi, L. Sainz, M. Chindris, "Steinmetz system design under unbalanced conditions", European Trans. on Electrical Power ETEP, Vol. 12, No. 4, pp. 283-290, 2002.

[13] D. Mayer, P. Kropik, "New approach to symmetrization of three-phase networks", Int. Journal of Electrical Engineering, Vol. 56, No. 5-6, pp. 156-161, 2005.

[14] F. de Leon, J. Cohen, "A practical approach to power factor definitions: transmission losses, reactive power compensation, and machine utilization", Proc. of the Power Eng. Soc. Meeting, IEEE DOI: 10.1109/PES.2006.1709175, 2006.

[15] W. G. Morsi, M. E. El-Hawary, "Defining power components in nonsinusoidal unbalanced polyphase systems: the issues", IEEE Trans. on PD., Vol. 22, No. 4, pp. 2428-2437, 2007.

[16] M. Malengret, C. T. Gaunt, "Active currents, power factor, and apparent power for practical power delivery systems", IEEE Access, Vol. 8, pp. 133095-133113, 2020.

[17] L. S. Czarnecki, "Currents' Physical Components (CPC) based Power Theory. A Review, Part I: Power properties of electrical circuits and systems", Przeglad Elektrotechniczny, Nr. 95, pp. 1-11, Nr. 10/2019.

[18] L. S. Czarnecki, "Currents' Physical Components (CPC) based Power Theory. A Review, Part II: Filters and reactive, switching and hybrid compensators", Przeglad Elektrotechniczny, R. 96, Nr. 4, pp. 1-11, 2020.

[19] D. A. Steeper, R. P. Stratford, "Reactive power compensation and harmonic suppression for industrial power systems using thyristor converters", IEEE Trans. on Ind. Appl., Vol. IA-12, No. 3, 232-254, 1976.

[20] J. E. Miller, Reactive power control in electric systems, John Willey \& Sons, 1982. 
[21] H. Akagi, Y. Kanazawa, A. Nabae, "Instantaneous reactive power compensator comprising switching devices without energy storage components", IEEE Trans. on Ind. Appl., IA-20, No. 3, pp. 625-630, 1984.

[22] N. Balabanian, Network Synthesis, Prantice-Hall, Englewood Cliffs, New York, 1958.

[23] L. S. Czarnecki, M. S. Hsu, "Thyristor controlled susceptances for balancing compensators operated under nonsinusoidal conditions", Proc. IEE, B, EPA, Vol. 141, No. 4, pp. 177-185, 1994.
[24] L. S. Czarnecki, "Do energy oscillations degrade the effectiveness of energy transfer in electrical systems," IEEE Trans. on Ind. Appl. DOI: 10.1109/TIA.2021.3051314, pp. 1-10, 2021.

[25] L. S. Czarnecki, M. Almousa and V. M. Gadiraju, "Why the Electric Arc Nonlinearity Improves the Power Factor of Ac Arc Furnaces?," 2018 IEEE International Conference on Environment and Electrical Engineering and 2018 IEEE Industrial and Commercial Power Systems Europe (EEEIC / I\&CPS Europe), Palermo, Italy, 2018, pp. 1-6, doi: 10.1109/EEEIC.2018.8493860. 